\title{
BRUGADA PATTERN IN HEROIN ADDICTION: SYNDROME OR PHENOCOPY?
}

\author{
Ettore Mancia ${ }^{1}$, Gregory Dendramis ${ }^{2}$, Caterina Carollo ${ }^{1}$, Laura Guarino ${ }^{1}$, Marco \\ Guarneri $^{1}$, Leonardo Calandra ${ }^{1}$, Santina Cottone ${ }^{1}$, and Giuseppe Mulè ${ }^{1}$ \\ ${ }^{1}$ Università degli Studi di Palermo \\ ${ }^{2}$ Ospedale Civico Palermo
}

August 20, 2020

\begin{abstract}
Introduction: Brugada phenocopies (BrPs) are clinical entities that differ in etiology from true congenital Brugada syndrome $(\mathrm{BrS})$ but have identical electrocardiographic (ECG) patterns. Methods: Our Manuscript reports a interesting case of BrP in a young man with heroin overdose who developed Acute Kidney Failure secondary to rhabdomyolysis. His initial ECG showed Brugada type 1 pattern with elevation "Coved-Type" of the ST segment in V1 and V2. The Brugada ECG pattern resolved upon hemodialysis treatment. Provocative testing using sodium channel blockers was performed, and failed to reproduce a BrS ECG pattern, confirming the diagnosis of Brugada's phenocopy. Conclusion: Several clinical conditions can imitate a true Brugada Syndrome and Brugada Phenocopies are a part of those conditions. A systematic approach and a prompt recognition of this clinical and electrocardiographic entity may avoid diagnostic errors and may promote the initiation of appropriate treatments.
\end{abstract}

\section{Hosted file}

Manuscript Journal Cardiovascular Electrophysiology-convertito.pdf available at https: //authorea.com/users/352572/articles/476752-brugada-pattern-in-heroin-addictionsyndrome-or-phenocopy

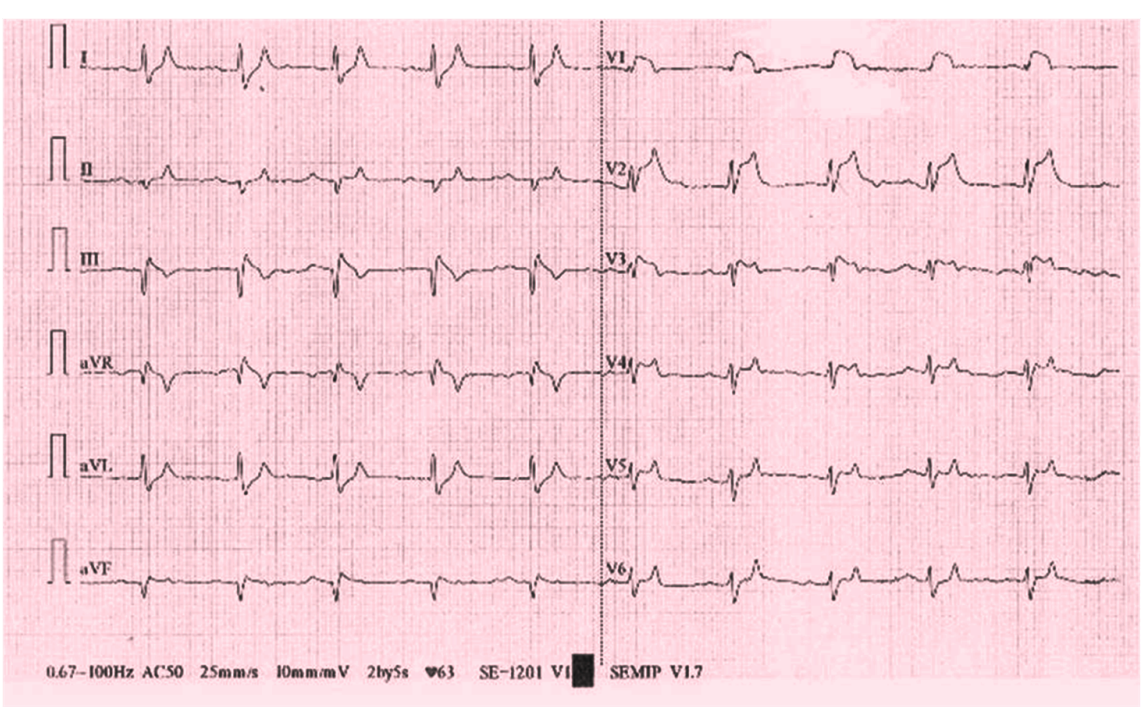




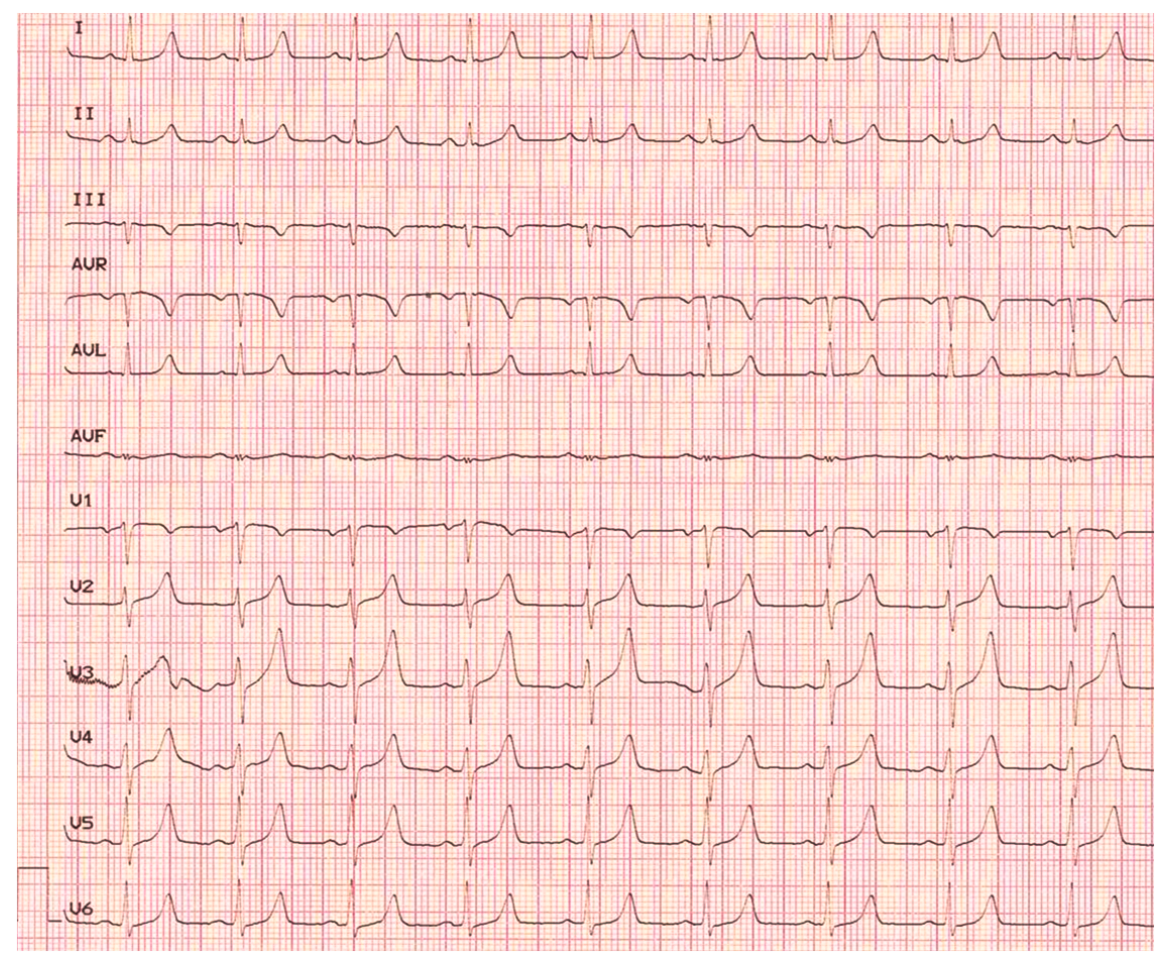




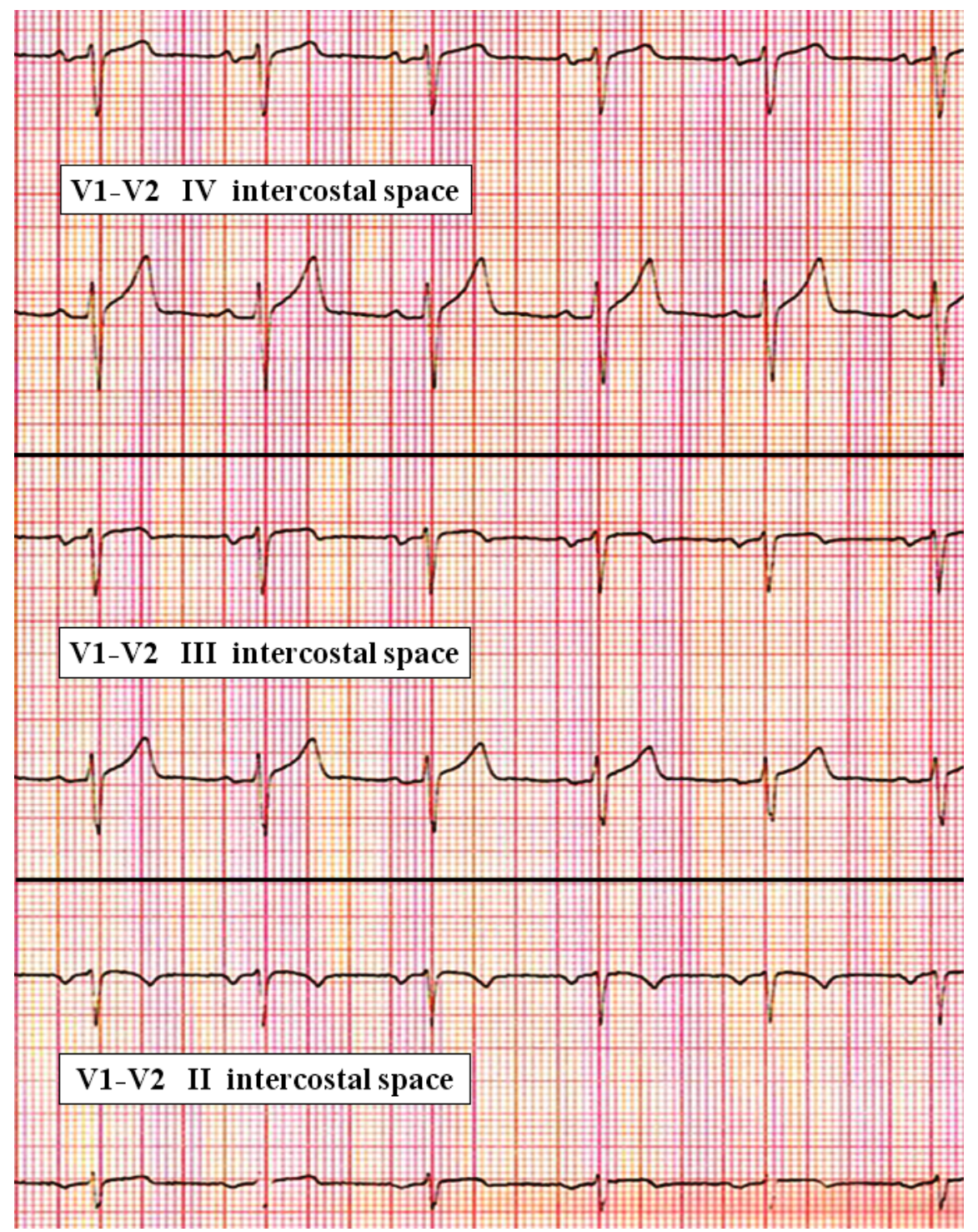

